\title{
El juego de rol como estrategia didáctica para el desarrollo de la conciencia ambiental. Una Investigación Basada en el Diseño.
}

\author{
Isabel María Cruz Lorite \\ Departamento de Didáctica de la Matemática, de las Ciencias Sociales y de las Ciencias \\ Experimentales. Facultad de Ciencias de la Educación. Málaga.imclorite@uma.es \\ ORCID:https://orcid.org/0000-0002-6099-134X
}

\section{María del Carmen Acebal Expósito}

Departamento de Didáctica de la Matemática, de las Ciencias Sociales y de las Ciencias Experimentales. Facultad de Ciencias de la Educación. Málaga. mcacebal@uma.es ORCID: https://orcid.org/0000-0002-5012-6459

\section{Daniel Cebrián Robles}

Departamento de Didáctica de la Matemática, de las Ciencias Sociales y de las Ciencias Experimentales. Facultad de Ciencias de la Educación.Málaga.dcebrian@uma.es ORCID: https://orcid.org/0000-0002-3768-1511

\section{Ángel Blanco López}

Departamento de Didáctica de la Matemática, de las Ciencias Sociales y de las Ciencias Experimentales.Facultad de Ciencias de la Educación.Málaga.ablancol@uma.es ORCID: https://orcid.org/0000-0003-3628-0801

[Recibido: 25 Septiembre 2019. Revisado: 15 Febrero 2020. Aceptado: 20 Abril 2020]

Resumen: Este trabajo muestra el desarrollo de experiencias de juegos de rol sobre la producción de energía nuclear a lo largo de tres cursos académicos, cuya metodología se ha ido modificando teniendo en cuenta los resultados obtenidos en cada una de ellas mediante una investigación basada en el diseño. Los sucesivos cambios metodológicos han mostrado mejoras en cuanto a los resultados obtenidos en cada experiencia respecto a las anteriores, concluyéndose que el juego de rol se muestra como una estrategia que favorece tanto el aprendizaje de conceptos científicos básicos como el tratamiento de aspectos concernientes a la conciencia ambiental, fomentando la práctica de habilidades argumentativas y poniendo en juego opiniones, creencias, actitudes y posiciones de muy diversa índole.

Palabras clave: juego de rol; investigación basada en el diseño; conciencia ambiental; profesorado en formación inicial

The role-playing game as a teaching strategy on environmental awareness development. A Design-Based Research.

Abstract: This work shows the development of role-playing game experiences on nuclear energy production over three academic years and whose methodology has been modified based on the results obtained in each of them, through a design-based research. The methodological changes have shown improvements in terms of the results obtained in each experience with respect to the previous ones, assuming the role-playing game as a strategy that favours the learning of basic scientific concepts, such as the treatment of aspects concerning environmental awareness, encouraging the practice of argumentative skills and implies opinions, beliefs, attitudes and positions of a very diverse nature.

Keywords: role-playing game, design-based research; environmental awareness; pre-service teachers 
Para citar este artículo: Cruz, I.M., Acebal, M.C., Cebrián, D. y Blanco, A. (2020) El juego de rol como estrategia didáctica para el desarrollo de la conciencia ambiental. Una Investigación Basada en el Diseño. Revista de Educación Ambiental y Sostenibilidad 2(1), 1302. doi: 10.25267/Rev_educ_ambient_sostenibilidad.2020.v2.i1.1302

\section{Introducción}

La búsqueda de mejoras docentes es una cuestión que suele discutirse en el ámbito de las políticas educativas en un nivel macro (recursos económicos, ratio, horas de clase, etc.), dejando al margen el nivel micro (Echeverría, 2010) del que forman parte las estrategias metodológicas que marcan el día a día en las aulas. El conocimiento didáctico del contenido de una determinada disciplina, en el que se incluye el de diferentes estrategias metodológicas (Valbuena, 2007), es uno de los componentes que constituyen lo que Rojas y Zapata (2017) denominan Conocimiento Profesional del Profesorado de Ciencias, del que forman parte también el conocimiento pedagógico, el conocimiento del contexto y el del contenido disciplinar. En este sentido, el uso de diferentes metodologías por parte de maestros y maestras en formación inicial (MFI en adelante), es una cuestión primordial para la mejora de su conocimiento didáctico de cara a su futuro profesional. No obstante, si bien es importante para los MFI tener conocimiento de la diversidad metodológica a su alcance, no lo es menos la toma de conciencia sobre la necesidad de evaluarlas, puesto que no hay dos grupos de estudiantes iguales y los contextos educativos cambian continuamente, tanto a nivel macro como micro.

El uso del juego de rol (JR en adelante) representa en la enseñanza en general (Matas, 2008), y en la de las ciencias en particular, una estrategia metodológica centrada en el debate (Colucci, Camino, Barbiero y Gray, 2006; Simoneaux, 2008), que permite la puesta en práctica y la mejora de las habilidades argumentativas de los participantes, a su vez consideradas necesarias para la mejora de la educación científica (Hodson, 2003). El JR se muestra adecuado, además, para el tratamiento de problemas sociocientíficos, como son los problemas ambientales (Matas, 2003; Rueda, 2018), y entre ellos el de la producción y el consumo de energía (Martín, Prieto y Jiménez, 2013). Uno de los debates sociales suscitados en los últimos años en España ha sido el concerniente a la producción de energía a través de centrales nucleares; cuestión en torno a la cual se han desarrollado los JR realizados durante esta investigación.

Usualmente se utilizan contextos de problemáticas ambientales para el desarrollo de la conciencia ambiental (CA en adelante) (p. ej. Karahan y Roehrig, 2015; Rueda, 2018; Puig y Evagorou, 2020). La CA se muestra como un constructo complejo, en el que confluyen varias dimensiones que atienden a aspectos cognitivos, afectivos, actitudinales y conductuales (Acebal, 2010). En esta investigación se parte de la existencia de relación entre la cultura científica y la CA (Muñoz, 2011; Sauvé, 2010), manteniendo la hipótesis de que la cultura científica, concebida como el conjunto de contenidos cognitivos y una visión de la ciencia, del mundo y de la manera de resolver los problemas que se enfrentan (Schein, 1988) compartida por todos los miembros de la sociedad, puede contribuir a generar CA, pues implica el reconocimiento del deterioro medio ambiental, sus causas y los riesgos asociados a él (Muñoz, 2011).

La investigación de la eficacia de las metodologías didácticas llevadas al aula debería ser una de las tareas más importantes del profesorado y de formadores y formadoras 
del profesorado. En este sentido, la investigación basada en el diseño (DBR, por sus siglas en inglés) (De Benito y Salinas, 2016) se muestra como el enfoque idóneo para estudiar el desarrollo de un JR mediante ciclos de mejora continuada en el que, de los resultados de una experiencia concreta, se obtiene la base para el diseño de la siguiente, y así sucesivamente.

\section{Marco teórico}

Según Gallego, Bustamante, Gallego, Salcedo, Gava y Alfaro (2017) está bien documentado que los conocimientos científicos y las creencias del profesorado sobre la ciencia tienen implicaciones sobre sus prácticas docentes (p. ej. Mansour, 2009), y que la relación entre las concepciones epistemológicas y el conocimiento pedagógico de este afecta al conocimiento profesional que va construyendo a la hora de desarrollar su práctica durante su trayectoria educativa. Así, continuando la reflexión de Gallego et al. (2017), se hace evidente la necesidad de modificar las ideas sobre la ciencia que poseen los MFI y se hace también necesario replantear las prácticas docentes usadas para tales fines.

Dada la situación actual y los grandes problemas ambientales a los que nos enfrentamos, la CA constituye uno de los aspectos que deben ser abordados en la formación del profesorado. Ahora bien, la CA es un concepto en constante construcción, cuya definición se presenta especialmente compleja, entre otros motivos, porque posee un amplio registro semántico, usado indistintamente en multitud de medios, áreas de conocimiento, sectores sociales y culturas, y refiriéndose, en muchas ocasiones, a cuestiones muy diferentes. Con independencia de la multitud de modelos existentes, muchos autores (p. ej. Jiménez y Lafuente, 2006; Acebal, 2010; Muñoz, 2011; Gomera, Villamandos y Vaquero, 2012) sostienen que la CA constituye la expresión final de la confluencia de, al menos, cuatro dimensiones: cognitiva, afectiva, conativa y activa, y que determina, en última instancia, el comportamiento y relación que el individuo establece con su medio ambiente.

Asumiendo que la cultura científica impregna, en mayor o menor medida, cada una de estas dimensiones, y considerando la íntima y multidimensional relación existente entre el medio ambiente y la ciencia (Muñoz, 2011), la mejora del aprendizaje de conocimientos científicos influiría en la CA. Un aspecto importante de la cultura científica lo constituyen las actitudes hacia la ciencia (Vázquez y Manassero, 2008) que están conformadas por diferentes factores entre los que se encuentra el conocimiento científico, que juega un papel importante en la construcción de la imagen y actitudes hacia la ciencia, como afirma (Muñoz, 2011). García, González, López, Luján, Martín, Osorio y Valdés (2001) señalaban que el conocimiento científico y tecnológico es necesario en la capacitación para la toma de decisiones o la comprensión de determinados puntos de vista sobre un problema sociocientífico. El tratamiento en la enseñanza de las ciencias de estos problemas sociocientíficos (Sadler, 2011) se considera hoy día un enfoque relevante para fomentar la cultura científica de los estudiantes y, por ende, mejorar su CA.

El uso del JR puede ser una estrategia metodológica apropiada para tratar los problemas sociocientíficos. Según Matas (2008) los juegos de simulación, entre los que se incluye el JR, se caracterizan por: a) incrementar el interés por aprender; b) precisar de una participación multidisciplinar; c) demandar la toma de decisiones; y 
d) estar orientados hacia el futuro, en tanto que dan la oportunidad al participante de tomar decisiones sobre problemas reales y observar las consecuencias más previsibles, así como de reorientar dichas decisiones en función de las retroalimentaciones recibidas. Entre los valores psicopedagógicos que aportan los juegos de simulación como estrategia metodológica, destacan su potencialidad para repasar conocimientos y habilidades, para el desarrollo del aprendizaje existente y el descubrimiento de aspectos deducibles a partir de este (Matas, 2008) y como elemento motivador (Matas, 2008; McSharry y Jones, 2000).

Como mencionan García et al. (2011), el JR, en tanto que juego de simulación, tiene como principal objetivo la representación de situaciones reales, defendiendo cada participante un papel e interactuando bajo las mismas reglas con el resto. Esta estrategia metodológica es comúnmente usada como medio para el estudio y promoción de las interacciones del alumnado por medio del diálogo basado en la argumentación (p. ej. Simonneaux, 2001; España, 2008; Juárez, Hierrezuelo, Cebrián y Franco, 2019). Permite la puesta en práctica de las habilidades argumentativas y la visibilización de un amplio abanico de puntos de vista que giran en torno a una determinada cuestión (España, Rueda y Blanco, 2013). La literatura al respecto muestra que el JR ayuda a mejorar los procesos de argumentación y refutación de opiniones contrarias (Simonneaux, 2008), además de fomentar la práctica de habilidades relacionadas con la expresión oral (Simonneaux, 2000), los procesos de resolución de problemas (España y Prieto, 2010), el trabajo colaborativo (Abella y Grande, 2010), el pensamiento crítico y el aprendizaje dialógico. Otras ventajas educativas del JR (España, Rueda y Blanco, 2013) son que presentan un contexto muy adecuado para que el alumnado experimente cambios de opinión (Simonneaux, 2001), así como la reflexión acerca de las propias opiniones, y explicite diferentes actitudes y valores (España, 2008). En relación a este último punto, el JR presenta también utilidad en el desarrollo de actitudes ambientales (Hernández, 2010).

El uso del JR en la formación inicial del profesorado es un área poco desarrollada, precisándose una mayor investigación e innovación (Rueda, 2018). No obstante, su uso en los programas de formación inicial es conveniente para que el futuro profesorado lo experimente desde la perspectiva del estudiante, lo que repercutirá en una mayor comprensión a la hora de utilizarlo en su enseñanza, así como en la probabilidad de hacerlo (Howes y Cruz, 2009). Este trabajo se centra en la investigación sobre el desarrollo y la mejora de un JR para el tratamiento de una cuestión sociocientífica y su posible impacto en la CA de MFI de educación primaria.

\section{Objetivos e hipótesis de investigación}

El objetivo general de la investigación es valorar si la mejora metodológica iterativa de un JR sobre energía nuclear consigue un mayor impacto en la CA de MFI de educación primaria.

Se establecieron como objetivos específicos los siguientes:

- Valorar si el nuevo diseño e implementación metodológica del JR mejora los resultados conseguidos en anteriores experiencias en cuanto al aprendizaje de contenidos científicos (dimensión cognitiva de la CA) y al cambio de posiciones respecto a problemas relacionados con la energía nuclear. 
- Valorar si el nuevo diseño e implementación metodológica del JR promueve el desarrollo de las demás dimensiones que conforman la CA (afectiva, conativa y activa)

La hipótesis inicial era que la mejora continua del diseño del JR redundaría en un mayor desarrollo de ciertos aspectos de la CA de los MFI; frente a la hipótesis nula, que plantearía que la mejora metodológica no tiene ningún efecto positivo.

\section{Metodología de investigación}

El diseño y desarrollo del JR se ha llevado a cabo mediante la DBR (de Benito y Salinas, 2016). La DBR tiene como fundamento la reivindicación de un nuevo enfoque metodológico que permita superar la brecha existente entre la investigación de los especialistas en didáctica y pedagogía y los problemas, necesidades e inquietudes que experimenta el profesorado y el alumnado en las aulas (Romero, 2014). Puede definirse, más que como un enfoque, como una serie de enfoques (Barab y Squire, 2004) cuya principal característica radica en la introducción de un elemento novedoso con intención de modificar una situación (De Benito y Salinas, 2016) y que presentan en común los procesos de prueba y revisión de cada elemento diseñado, en el que las sucesivas iteraciones (diseño, implementación y revisión) desempeñan un papel similar al de la variación sistemática en el experimento (Cobb, diSessa, Leher y Schauble, 2003). Los cambios realizados en cada ciclo de revisión se comentan en el siguiente apartado, donde se describe la propuesta didáctica llevada a cabo. Las diferentes implementaciones de esta estrategia llevadas a cabo en la formación inicial de profesorado se han realizado mediante el estudio de casos múltiples con intención comparativa, o multicaso, de tipo analítico con hipótesis (Tójar, 2006) en los que cabe considerar como grupo de control a aquel con el que se realizó la primera implementación. En la DBR suelen recogerse gran cantidad de datos de distinta naturaleza. Por esta razón, para el análisis de los mismos se ha adoptado un enfoque de investigación mixto (Creswell, 2005). En concreto, los datos analizados en este trabajo corresponden tanto a preguntas cerradas como abiertas.

\section{Participantes}

En la primera experiencia participaron $32 \mathrm{MFI}$, en la segunda 93 y en la tercera 78. Todos cursaban la asignatura de Enseñanza de las Ciencias de 3o de Grado en Educación Primaria en la Facultad de Ciencias de la Educación de la Universidad de Málaga. En general, comprendían edades entre los 20 y los 21 años, con un perfil de estudios previos situado en el ámbito de las ciencias sociales y humanidades, y habían cursado asignaturas científicas de forma reglada por última vez en educación secundaria obligatoria.

\section{Instrumentos de recogida de datos}

Durante la investigación se utilizaron cuestionarios a modo de pretest (Pre en adelante) y postest (Pos en adelante), hojas de registro (utilizadas antes y durante el juego) y grabaciones en vídeo y audio de las escenificaciones. En este trabajo se presentan solo los resultados obtenidos a partir de los cuestionarios.

El cuestionario fue modificado en cada experiencia: en la primera experiencia (Anexo I) el Pre contaba con 9 preguntas y el Pos con 11, en la segunda experiencia (Anexo II) el Pre tenía 11 preguntas y el Pos 17 y en la tercera experiencia (Anexo III) el Pre 
tenía 16 preguntas y el Pos 17. Los diseños de las dos primeras versiones del cuestionario se centraron fundamentalmente en el registro de conocimientos básicos sobre energía nuclear y en cuestiones de tipo afectivo, para las que debía tomarse una postura acerca del dilema tratado. Incluían también, sobre todo la segunda versión, preguntas sobre aspectos didácticos, para conocer el grado de utilidad que los MFI percibían del uso del JR como estrategia didáctica para la educación primaria. En la tercera versión, además de mantener las preguntas relacionadas con conocimientos, aspectos afectivos y de tipo didáctico, se introdujeron cuestiones relativas a las dimensiones conativa y activa: preguntas P12, P13, P14, P15 y P16 en Pre y Pos. Este bloque de preguntas fue formulado haciendo referencia al consumo energético en general, dada la dificultad que supondría su formulación referida sólo a la energía nuclear. Las P12 y P13, referentes a aspectos de la dimensión afectiva de la CA, consultaban al alumnado acerca de la importancia que tiene el conocimiento de las fuentes de las que proviene el consumo energético cotidiano. La P14 (dimensión conativa), versaba sobre la predisposición a asistir a eventos de diversa índole para informarse sobre dicho consumo energético. La P15 del Pre (dimensión activa), preguntaba sobre conductas habituales que los alumnos pudieran tener en cuanto al uso responsable de la energía. Esta pregunta fue formulada de forma diferente en el Pos, pero numerada también como P15, y con ella se pretendía que los MFI expresasen la nueva información adquirida acerca de prácticas y conductas cotidianas para desarrollar un uso más responsable de la energía, atendiendo en este caso más a aspectos cognitivos. La P16 del Pre (que no tiene equivalente en el Pos y tenía un carácter conductual) solicitaba información acerca de la participación, pasada o presente, en eventos de diferente índole dirigidos a la información y formación sobre el consumo energético. En las P15 y P16 del Pre se consultaba al alumnado sobre sus conductas, lo que supone una fuente de datos indirecta. La dimensión activa de la CA es la que en menor medida podría ser atendida, tanto en cuanto a su evaluación como a la medición de un posible cambio, dada la imposibilidad de observar de forma directa las conductas del alumnado. Los datos obtenidos son, por tanto, información acerca de la conducta autopercibida. El orden establecido para las preguntas del cuestionario también fue modificado respecto al de los cuestionarios anteriores. El contenido del cuestionario fue validado mediante juicio de expertos (Lacave, Molina, Fernández y Redondo, 2016), en concreto por dos profesores e investigadores con amplia experiencia en educación ambiental.

\section{Análisis de los datos}

Se utilizó el programa de análisis cualitativo NVivo. v12, con el que se realizó el análisis y categorización de las preguntas abiertas del cuestionario. Las preguntas cerradas fueron analizadas con el software SPSS. v15. Para el análisis de posibles diferencias significativas, al ser la distribución de los datos no normal, se utilizaron las pruebas no paramétricas para muestras relacionadas de McNemar (variables dicotómicas), prueba de rangos de Wilcoxon (variables politómicas ordinales) y el coeficiente Chi-cuadrado ${ }^{1}$ mediante tablas de contingencia (variables politómicas nominales) para estudiar las diferencias Pre-Pos; y la prueba U de Mann-Whitney

\footnotetext{
${ }^{1}$ Se utilizó la prueba exacta de Fisher para aquellos casos en los que más del 25\% de las casillas de la tabla tenían frecuencias menores a 5 y se estableció un nivel de significación de 0.05 para todas las pruebas.
} 
(variables politómicas ordinales) para muestras independientes con el fin de comparar entre sí los subgrupos participantes en cada experiencia.

A la calificación de "respuesta adecuada" siempre se alude desde el punto de vista del conocimiento científico.

\section{Diseño y desarrollo del juego de rol mediante la DBR}

De acuerdo con el enfoque de la DBR, el diseño y desarrollo de este JR se ha llevado a cabo mediante tres ciclos de diseño, implementación y evaluación. A continuación, se describe cada uno de estos ciclos, resaltándose los cambios que se realizaban de un ciclo a otro en función de los resultados obtenidos en la evaluación.-

\section{Primer ciclo}

En este primer ciclo (curso 2015/16), el diseño inicial del JR se llevó a cabo partiendo de estudios anteriores realizados por miembros del grupo de investigación en Enseñanza de las Ciencias y las Competencias (ENCIC) de la Universidad de Málaga (España, 2008; España, Rueda y Blanco, 2013; Cebrián, España y Blanco, 2018).

En este ciclo, el escenario del JR se centró en la posibilidad de realizar un cambio legislativo para ampliar el periodo de vida útil de la central nuclear de Garoña (Villa, 2016), situación a la que tuvo que enfrentarse el gobierno de aquel momento. Se trataba de otorgar relevancia y actualidad al problema tratado. En esta primera implementación los MFI se organizaron en pequeños grupos en los que cada uno debía defender un rol. Había un total de 10 roles, 5 a favor de la ampliación del periodo de vida útil de la central de Garoña (científico nuclear, ciudadano de Garoña 1, diputado del Gobierno, lobby nuclear y trabajador de la central) y 5 en contra (científico en contra, ciudadano de Garoña 2, diputado de la oposición, ecologista y empresario de una gran hidráulica), que debían debatir acerca del posible cambio legislativo, y 1 moderador. Durante el juego, ninguno de los participantes debía revelar su identidad ficticia, de manera que el objetivo final del juego era el de averiguar qué rol defendía cada uno de ellos. El juego se desarrollaba en una única ronda de intervenciones en la que cada rol debía lanzar sus pruebas. En esta implementación no se solicitaba la elaboración de argumentos completos, sino que, dada la duración establecida para los juegos de rol, cada participante debía aportar pruebas, que se definen como aquello a lo que apelamos con el fin de mostrar que un determinado enunciado es cierto o es falso (Jiménez-Aleixandre, 2010). Estas pruebas podían estar constituidas por información que podía ser tanto cuantitativa (p. ej. magnitudes, cantidades o relaciones) como cualitativa. Mientras, el resto de roles debían anotar las pruebas del resto para intentar averiguar qué rol defendía cada uno. La duración estimada de la escenificación del JR era de 30 minutos y los MFI dispusieron de una semana para su preparación.

\section{Segundo ciclo}

El problema en torno al cual giraba el debate varió en cada ciclo, de acuerdo a la evolución del debate social real en el cual estaba basado. Así, en este segundo ciclo (curso 2017/18), y con la central de Garoña ya cerrada (Cervilla, 2017), se debatió acerca de la campaña planteada por Greenpeace augurando el cierre del parque nuclear español para el año 2020 (RTVE, 2017). 
Con respecto al primer ciclo, también se propuso como mejora la elaboración de argumentos y contrargumentos, no centrándose solo en pruebas. Se propuso como contexto del juego la simulación de un programa televisivo. Otra modificación fue la división de cada clase en dos subgrupos, en los que a su vez se formaron pequeños grupos, tantos como roles tuviera el juego. En cada rol, uno de los integrantes hacía de portavoz, mientras que el resto formaba el "equipo asesor", encargado de prestar apoyo al portavoz durante el debate ofreciéndole información con la que contrargumentar a los demás roles. Se asignaron 4 roles a favor de la campaña de Greenpeace (científica a favor, ciudadana, ecologista y empresario de una gran hidráulica) y 4 roles en contra (científica nuclear, propietario de la central nuclear clausurada de Garoña, político del gobierno, antiguo trabajador de la central nuclear clausurada de Garoña), además del presentador. La escenificación del JR tuvo una duración media de una hora, y los MFI dispusieron de una semana para su preparación. En esta segunda experiencia se procuró alternar el sexo de la nomenclatura y descripción de los roles, dado que en la primera experiencia todos habían sido nombrados y descritos en masculino, lo que se consideró que podía lanzar un mensaje poco igualitario en lo que a cuestión de género se refiere. Durante la rueda de presentación, el equipo asesor debía registrar los argumentos débiles y fuertes de los roles contrarios. Se otorgaba un tiempo de 5 a 6 minutos (corte publicitario) para que los portavoces se reunieran con sus equipos y elaboraran los contrargumentos para la segunda parte de debate. En el debate los portavoces debían contrargumentar los argumentos iniciales del resto. Al terminar, el equipo de prensa del programa (equipo asesor del presentador) emitía una respuesta a la pregunta inicial del debate a partir de los resultados de las valoraciones y votaciones del público (los participantes del JR podían interactuar a través de herramientas TIC enviando mensajes cortos que se proyectaban en una pantalla grande, a modo de opiniones del público, al igual que votar al rol que más les hubiera convencido).

\section{Tercer ciclo}

En el tercer y último ciclo, la cuestión abordada fue el pacto acordado por algunas empresas energéticas en cuanto al cierre progresivo del parque nuclear español, que culminaría entre los años 2030 y 2035 (RTVE, 2019). En la tercera implementación se mantuvo el esquema general utilizado en las dos anteriores, pero se dio un mayor espacio al tratamiento de aspectos afectivos y actitudinales, así como al análisis de ciertos aspectos conductuales, desde el punto de vista ambiental, sobre la cuestión energética. Así, se cambiaron algunos roles y su descripción con objeto de complicar la dinámica, sobre todo en cuanto a la práctica de las habilidades argumentativas y la profundización en las diferentes sensibilidades y visiones que podía haber respecto al problema tratado. Si bien los perfiles de los roles utilizados hasta ahora defendían diferentes intereses (por ejemplo, el ecologista tenía únicamente motivaciones ambientales mientras que las del empresario de la gran hidroeléctrica eran económicas), todos defendían una posición radical frente al uso de energía nuclear (tanto el ecologista como el empresario de la gran hidroeléctrica estaban totalmente en contra de su uso).

En esta tercera experiencia, los perfiles fueron redefinidos, de tal forma que un rol podía estar en contra del uso, pero a favor del almacenamiento de residuos, o a favor de su uso, pero solo durante cierto periodo de tiempo, caracterizándose así de forma más compleja cada personaje. Se establecieron 5 roles más afines al pacto realizado 
por las empresas energéticas (científico/a de energías renovables, ciudadano/a del pueblo, ecologista, empresario/a de energía solar y político/a de la oposición) y 5 menos afines (científico/a nuclear, político/a del gobierno, promotor/a de un cementerio nuclear, propietario/a de una central nuclear y trabajador/a de una central nuclear) y la escenificación del JR tuvo una duración media de una hora. Se realizó una primera sesión para explicar el JR: escenario, personajes, reglas y formación de los pequeños grupos que defenderían los diferentes roles, previa cumplimentación de un cuestionario para conocer los conocimientos sobre las centrales nucleares y aspectos sobre la CA. Tras dejar una semana para la preparación de los roles, en una segunda sesión se realizaba la escenificación. Para la primera parte, el portavoz del rol presentaba los argumentos iniciales durante un tiempo máximo de tres minutos, sin que nadie pudiera intervenir; mientras, el resto de participantes del grupo anotaban las debilidades y fortalezas de los argumentos del resto de roles para elaborar contrargumentos. Durante la publicidad el equipo asesor presentaba y abordaba con el portavoz los contrargumentos elaborados. Finalmente, y como novedad, en la ronda de debate cada uno de los roles utilizaba los contrargumentos elaborados para rebatir al resto de roles, al tiempo que redactaban refutaciones a las contrargumentaciones que recibían por parte del resto. Como en la anterior experiencia, el equipo de prensa emitía una valoración general del debate. Finalmente, los MFI contestaban el mismo cuestionario para conocer si había habido un cambio en conocimientos, actitudes y posición frente a la pregunta en cuestión.

\section{Resultados}

\section{Sobre el conocimiento científico}

Para poder comparar unas experiencias con otras, se han analizado preguntas que, por el conocimiento que requerían poner en juego, se consideran contrastables. La correspondencia entre estas, era: pregunta 1 de los tres cuestionarios (Anexos I, II y III), denominada en el comentario de los resultados P1; preguntas 3 (Anexos I y II) y 9 (Anexo III), denominadas P2; y preguntas 5 (Anexo I), 6 (Anexo II) y 8 (Anexo III) denominadas P3.

Para P1, la tendencia en las dos primeras experiencias fue muy similar, tanto entre Pre y Pos como entre una experiencia y otra (Figura 1), la mayor parte contrarias a la energía nuclear.

En la tercera experiencia se registró una disminución del porcentaje de MFI que se posicionaban a favor de la producción nuclear de forma indefinida respecto a las experiencias anteriores (10\% en Pre y Pos). Si bien en el Pre las posiciones se encontraban divididas a partes iguales entre aquellos que estaban de acuerdo con el pacto y aquellos que apostaban por el cierre total del parque nuclear para el año 2020 ( $45 \%$ en ambas), en el Pos se movilizaron algunas opiniones de forma favorable al acuerdo con el pacto (56\%). 


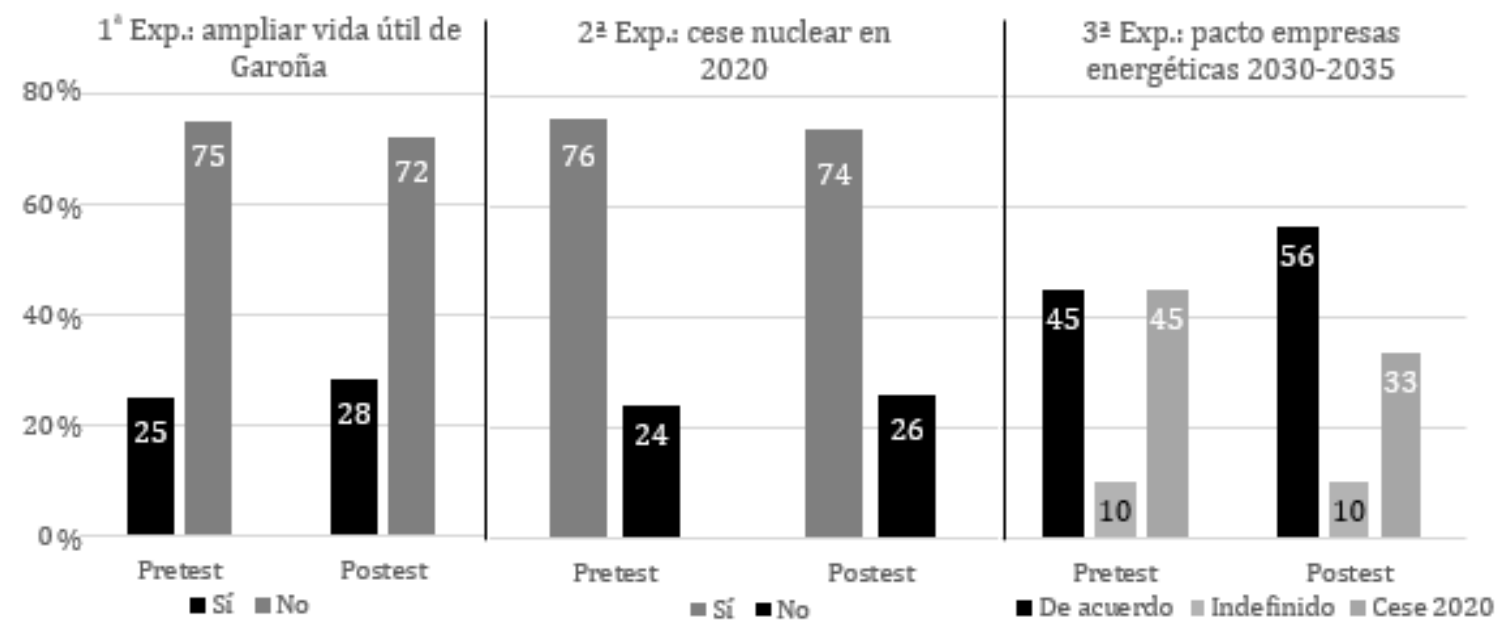

Figura 1. Porcentajes de respuesta obtenidos para P1 en cada experiencia.

En la P2 (Figura 2) sobre las emisiones producidas por las centrales nucleares, el cuestionario utilizado en la primera experiencia contemplaba la respuesta múltiple, por lo que para poder comparar los datos con los de experiencias posteriores, estas se agruparon en respuestas adecuadas ${ }^{2}$ ("agua" o "vapor de agua") e inadecuadas (el resto). No obstante, para la primera experiencia se mantuvo una categoría más, que comprendía los casos que habían marcado "agua" y otra u otras opciones. En cuanto a las diferencias entre Pre y Pos, en la primera experiencia se registró un incremento del 41\% de respuestas adecuadas; además de no registrarse en el Pos ningún caso en que se marcase "agua" y otra opción de forma conjunta, siendo estas diferencias estadísticamente significativas $(\mathrm{Z}=-2.651$; $\mathrm{p}$-valor=0.008). En la segunda experiencia dicho aumento fue prácticamente el mismo (40\%), aunque el porcentaje de alumnos que no contestaban o no sabían la respuesta pasó del $16 \%$ al $1 \%$. Se encontraron también diferencias estadísticamente significativas $(Z=-5.689 ; p$-valor=0.000). En la tercera experiencia el aumento de respuestas adecuadas se redujo a la mitad (20\%) que el de las experiencias anteriores. Aunque se redujo el porcentaje de alumnos que no contestó a la pregunta (del $26 \%$ al $8 \%$ ), el de alumnos que marcaron opciones no adecuadas se mantuvo prácticamente inalterado (62\% en el Pre y $59 \%$ en el Pos). No obstante, las diferencias halladas, como en el resto de experiencias, son estadísticamente significativas $(\mathrm{Z}=-3.633$; $\mathrm{p}$-valor=0.000).

\footnotetext{
${ }^{2}$ A la calificación de "respuesta adecuada" siempre se alude desde el punto de vista del conocimiento
} científico. 


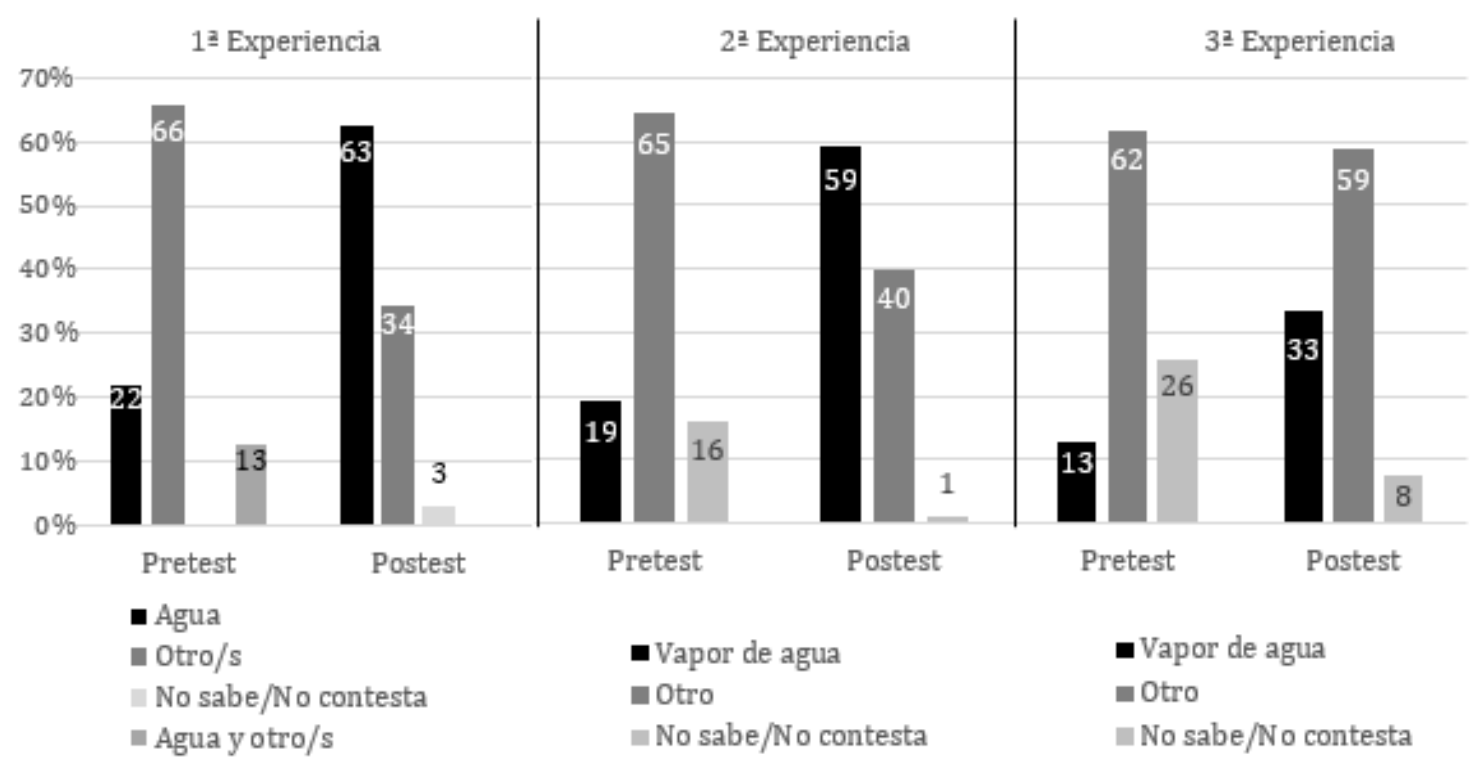

Figura 2. Porcentajes de respuesta obtenidos para P2 en cada experiencia.

$\mathrm{Al}$ analizar por subgrupos (correspondientes a dos clases de la misma asignatura) los datos de la tercera experiencia, se observó que uno de ellos registraba una tendencia inversa a la que se venía produciendo en experiencias anteriores, con un aumento de respuestas inadecuadas en el Pos (78 \%) respecto al Pre (57\%). Mientras que un subgrupo experimentó un aumento del $34 \%$ de alumnos que reconocían el vapor de agua como única emisión gaseosa directa de las centrales nucleares, en el otro subgrupo el aumento fue solo del $5 \%$. Además, en este último se observó un aumento del porcentaje de alumnos que marcó la opción de "no expulsan gases" entre Pre (16 \%) y Pos (35 \%), y la disminución del porcentaje de alumnos que no contestaron, del $32 \%$ al $5 \%$. El resto de porcentajes se mantuvo prácticamente igual. De las grabaciones en vídeo pudo extraerse que en ningún momento salió a debate qué sustancia era exactamente la que emitían las centrales nucleares. Se encontraron diferencias estadísticamente significativas entre los Pos de ambos subgrupos ( $\mathrm{Z}=-$ 2.368; p-valor $=0.018$ )

En cuanto a la P3, en la primera, la segunda y la tercera experiencia se produjo un aumento del porcentaje de alumnos que marcaron "fisión nuclear" como único proceso realizado en las centrales nucleares, de un $3 \%$, un $26 \%$ y un $37 \%$ respectivamente (Figura 3), registrándose el mayor aumento, por tanto, con la tercera propuesta metodológica. Las pruebas estadísticas mostraron diferencias significativas Pre-Pos para la segunda $(\mathrm{Z}=-4.350$; $\mathrm{p}$-valor $=0.000)$ y la tercera experiencia $(Z=-4.190$; $p$-valor=0.000). 


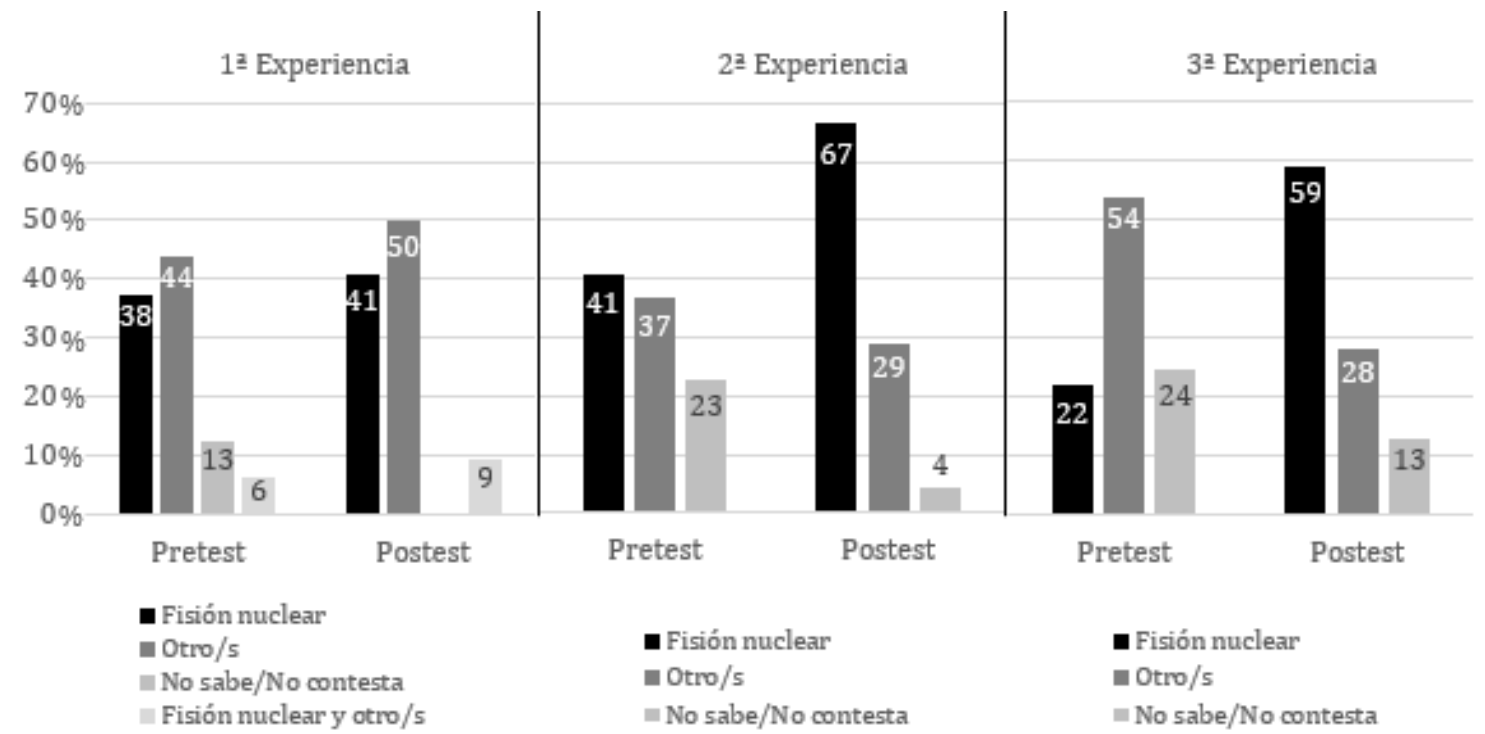

Figura 3. Porcentajes de respuesta obtenidos para P3 en cada experiencia.

\section{Sobre otros aspectos de la conciencia ambiental}

A continuación, se muestran los resultados de algunas de las preguntas que se incluyeron en la tercera versión del cuestionario con la intención de poder obtener información acerca de más aspectos concernientes a la CA que en las versiones anteriores.

La P13, en la cual los MFI debían justificar la importancia que daban a informarse acerca del tipo de fuentes de las que procede el consumo energético cotidiano, mostró que la mayor parte de las justificaciones realizadas tanto en el Pre (40\%) como en el Pos (34\%) se referían a aspectos ambientales (Tabla 1). Tras estas, el segundo código más identificado, también tanto en el Pre (28 \%) como en el Pos (33\%) era el relativo al conocimiento; estas respuestas redundaban en la formulación de la propia pregunta, es decir, la justificación dada a la importancia de informarse acerca del consumo era simplemente la importancia de adquirir conocimiento, sin especificar exactamente para qué. Los cambios más significativos fueron los registrados para los códigos etiquetados como actuar y perjuicios. El porcentaje de alumnos que justificaba la importancia de informarse aludiendo a los posibles perjuicios derivados de su uso, sin especificar cuáles eran estos perjuicios, pasó de un $9 \%$ en el Pre a un 2 $\%$ en el Pos. En cuanto a las justificaciones que apoyaban la importancia de informarse para poder actuar (colectiva o individualmente) aumentaron de un $5 \%$ en el Pre a un $13 \%$ en el Pos.

Se han comparado las respuestas del Pre y del Pos de la P15 (Figura 4), para estudiar las posibles diferencias entre las conductas habituales que los MFI habían dicho tener en cuanto al uso responsable de la energía con las nuevas prácticas que hubiesen pensado o descubierto tras la realización del JR. De las categorías creadas, la mayor parte de los MFI dijeron que sus conductas habituales (P15 del Pre) eran la reducción del consumo (21\%), apagar las luces de las estancias del hogar (19\%), aprovechar las horas de luz (13\%) y desenchufar diferentes aparatos y electrodomésticos cuando no están siendo usados (12\%). El $9 \%$ dijo no realizar ninguna práctica cotidiana en este sentido y el $4 \%$ no saber cómo contribuir de forma particular al uso responsable de la energía. 
Tabla 1. Frecuencias y porcentajes de las categorías creadas para el análisis de la P13 en el Pre y el Pos de la tercera experiencia.

P13 Pre P13 Pos

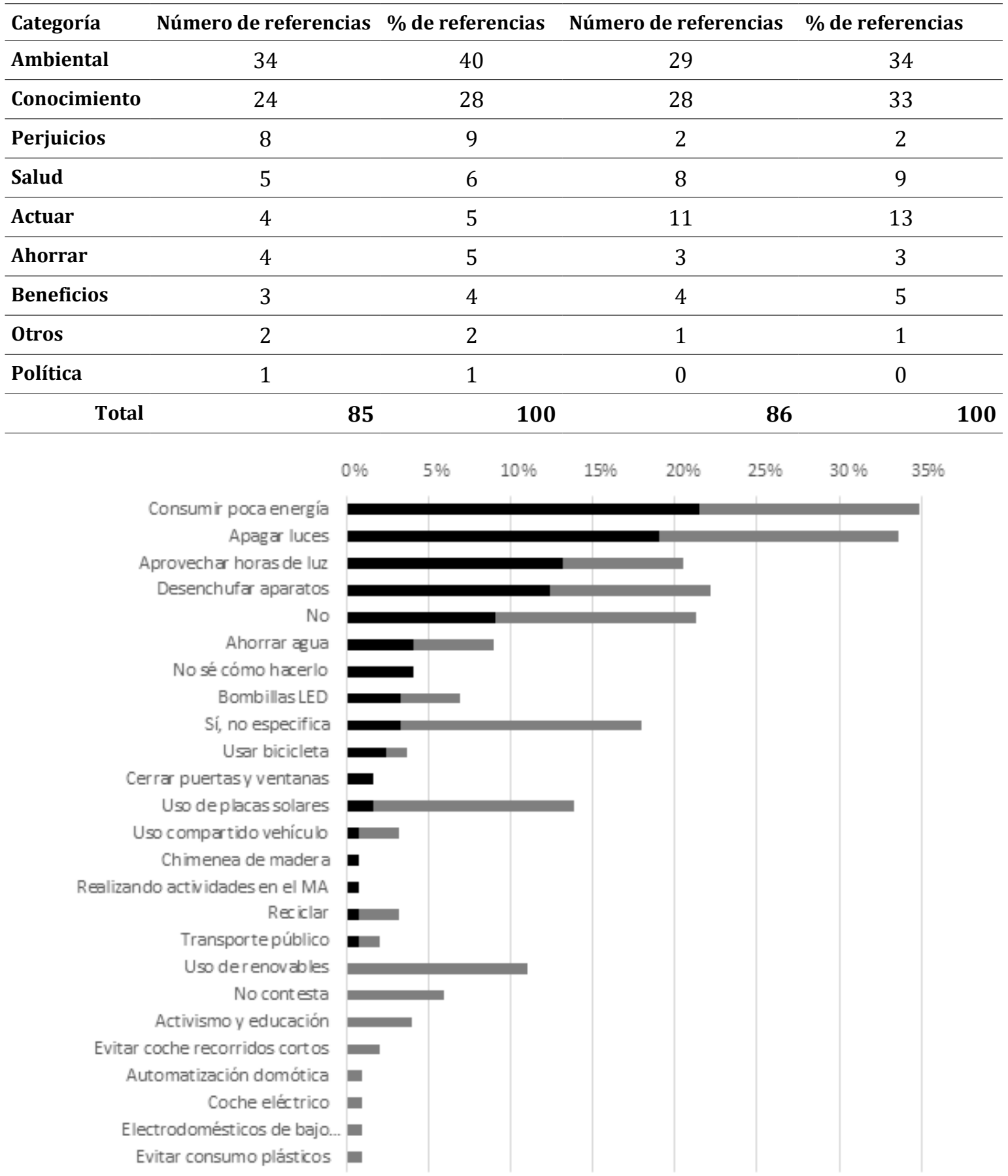

P15, pretest: ¿Contribuyes o intentas contr buir con tus conductas hab tuales para hace un uso responsable de la ener gía? Si es así, ¿ de qué forma?

- P15, postest: Durante la prepa ación del juego de rol, ¿ has descubier to o pensado medidas con las que podría contribuir con tus conduct as habituales para hacer un uso responsable de la ene gía? Si es así, ¿cuál/es?

Figura 4. Porcentajes de respuesta obtenidos para la P15 (Pre y Pos) de la tercera experiencia.

Como prácticas nuevas que hubiesen conocido tras el JR (P16 del Pos), y coincidiendo tres de ellas con las categorías más referidas en la pregunta del Pre, la reducción del 
consumo (11\%), apagar luces innecesarias en el hogar (12\%), desenchufar aparatos (8\%) y el uso de placas solares (10 \%) fueron las prácticas más comentadas. Esta última categoría aumenta de forma considerable tras la realización del JR. Por otro lado, ningún alumno menciona no tener información sobre qué prácticas poder realizar; no obstante, hay un $12 \%$ de alumnos que manifiestan no haber descubierto ninguna práctica nueva, otro $12 \%$ decían conocer nuevas prácticas de uso responsable de la energía, aunque no especificaban ninguna en concreto, y un $5 \%$ no contestaron. Sin embargo, surgen ideas nuevas en las respuestas analizadas en el Pos. Si bien estas categorías no se encuentran representadas con mucha frecuencia, muestran cierto aumento en cuanto al rango de prácticas consideradas por los MFI, como la automatización domótica (1\%), el uso de coches eléctricos (1\%), evitar hacer uso del vehículo particular para recorridos cortos (1\%), utilización de electrodomésticos de bajo consumo (1\%), evitar el consumo de plásticos (1\%) y el activismo y la educación (4\%). En esta última, varios casos se refieren a la utilidad del activismo como instrumento de presión hacia los poderes gubernamentales y, en cuanto a la educación, a su futuro profesional como docentes de educación primaria.

Respecto a las preguntas del Pre relacionadas con el interés sobre el consumo energético (Figura 5), en la P12, el $100 \%$ de los alumnos consideró importante informarse acerca del tipo de fuentes del que procede su consumo energético cotidiano tanto en el Pre como en el Pos. En la P14, el 95 \% y el $94 \%$ de los alumnos afirmó que estaría dispuesto a asistir a eventos relacionados con la información acerca del consumo energético en Pre y Pos, respectivamente. Por último, en la P16, acerca de las conductas actuales o pasadas en relación al uso responsable de la energía, el $82 \%$ de los alumnos dijo no haber asistido a eventos, ni haberse informado sobre el consumo energético. El $8 \%$ decía informarse o haberse informado en los medios de comunicación masivos y un $5 \%$ manifestaba haber asistido a charlas y manifestaciones, aunque de temática ambiental en general.

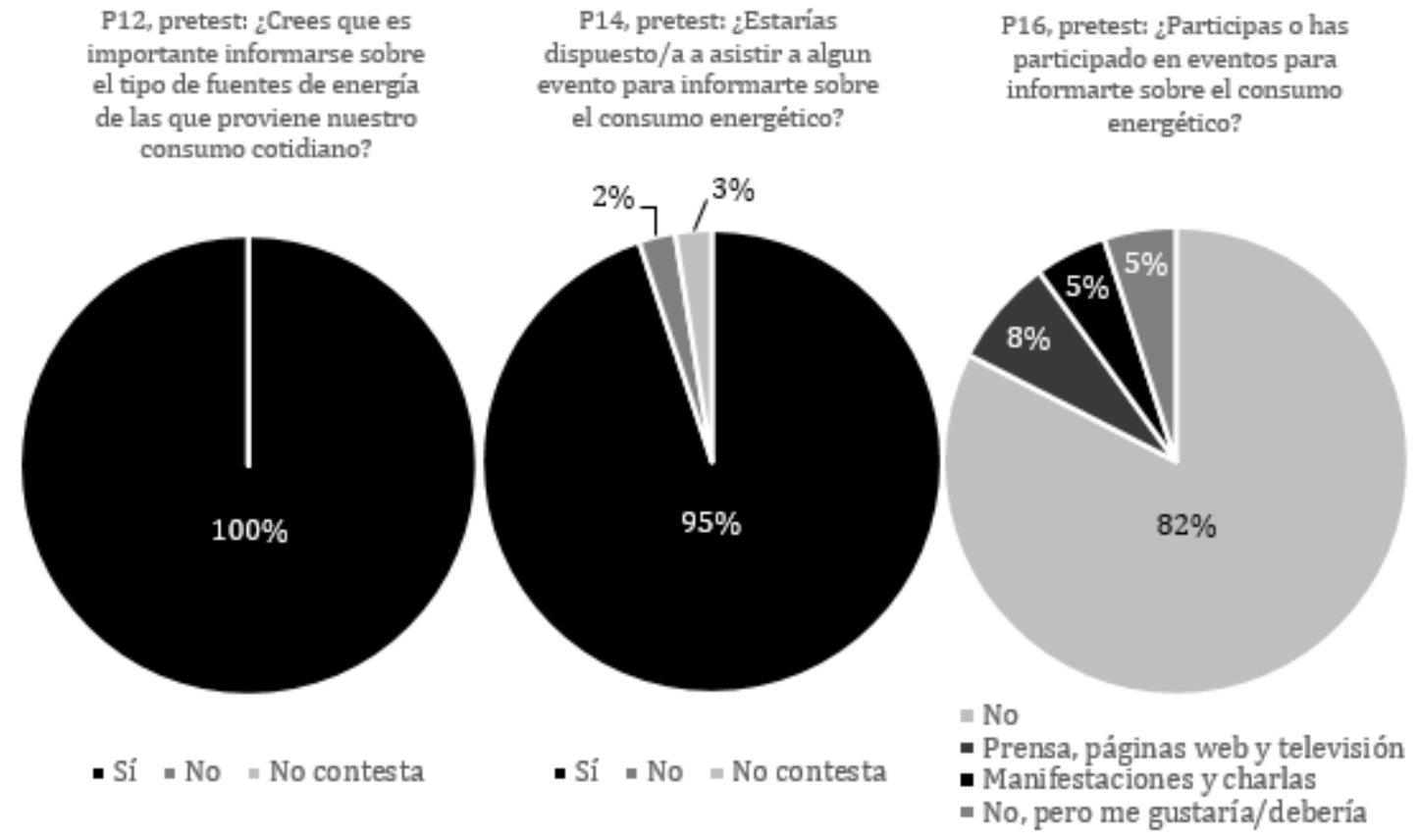

Figura 5. Porcentajes de respuesta obtenidos para P12, P14 y P16 en el Pre de la tercera experiencia. 


\section{Discusión y conclusiones}

Atendiendo a los objetivos fijados al principio de este trabajo y a los resultados obtenidos, puede afirmarse que la DBR ha resultado útil en el proceso de mejora del JR, sobre todo en cuanto a la mejora del tratamiento de los procesos que producen la movilización de posiciones en torno a un problema concreto y el aprendizaje de conocimientos científicos básicos acerca de la producción de energía nuclear.

Con la tercera experiencia se ha logrado producir un cambio de posiciones de los participantes con respecto al pacto de cierre del parque nuclear, cuestión que no se producía en las dos experiencias anteriores. Consideramos que esto puede deberse a la apertura del rango de opciones disponibles en P1 que permitió visualizar de forma más clara el cambio de posiciones entre Pre y Pos. Entendemos este como un resultado positivo, dada la importancia de crear contextos en los que el futuro profesorado pueda experimentar cambios de opinión. Esta es una de las posibilidades que ofrece el JR, dadas las dificultades que estos encuentran a la hora de cambiar sus posiciones para convertirse en gestores del debate en el aula, en lugar de mostrarse como "los que saben" (Simonneaux, 2001), es decir, no adoptar un rol de experto o autoridad en el tema debatido, sino el de guía y moderación.

Cada modificación del JR parece haber producido mejoras en el aprendizaje de conocimientos científicos. Los resultados obtenidos para P3 muestran claramente cómo la diferencia entre las respuestas adecuadas entre Pre y Pos se ha incrementado en cada experiencia, experimentándose la mayor mejora en la segunda experiencia. No sucede así en el caso de P2, consiguiéndose porcentajes de respuestas similares en la primera y segunda experiencia, y disminuyendo en la tercera. Respecto a este hecho, la hipótesis principal atiende en mayor medida a la formulación de la pregunta y a aspectos metodológicos del JR. No obstante, al estudiarse las características intrínsecas del grupo se observa que, de las tres experiencias, el grupo de estudiantes de la tercera es el que partía de un menor porcentaje de respuestas adecuadas, tanto para P2 como para P3. Por otro lado, ha podido contrastarse, partiendo de las grabaciones en vídeo que se hicieron de las escenificaciones de todos los JR y de la percepción de los investigadores que estuvieron presentes durante su realización, que en ningún momento salió a debate cuál era exactamente la sustancia emitida por las centrales nucleares. Además, los roles posicionados a favor de la energía nuclear fueron especialmente insistentes en la defensa de que las centrales nucleares no contaminaban, lo que pudo crear, en algunos MFI que no habían contestado en el Pre o decían no saber la respuesta (ya que el porcentaje que contestó "dióxido de carbono" se mantuvo), la falsa idea de que las centrales no tenían ningún tipo de emisión gaseosa. Esto implica la necesidad de revisar la última propuesta metodológica, al no haberse contemplado (tampoco en las experiencias anteriores) las necesidades particulares que pudiera tener algún grupo concreto, y la especial atención que esto requeriría; atenciones como pudieran ser la ampliación del periodo de preparación del JR, o la mayor definición de los personajes, especificando aún más, por ejemplo, los temas que su rol, como experto, debe controlar de cara al debate. Rueda (2018) sugería la evaluación del impacto de los programas formativos que incluyen actividades de JR sobre el conocimiento científico del profesorado en formación inicial, por lo que, como conclusión acerca de este aspecto, consideramos que este trabajo supone una contribución relevante a dicha línea de investigación, puesto que ha mostrado resultados positivos. 
En cuanto al segundo de los objetivos, asumiendo que la relación entre actitudes medioambientales y conducta es más fuerte cuanto mayor es el conocimiento sobre el medioambiente (Meinhold y Malkus, 2005), puede decirse que el JR ha contribuido a desarrollar la dimensión cognitiva de la CA de los MFI, aunque no puede afirmarse lo mismo respecto al resto de dimensiones que la componen. Respecto a las preguntas finales del cuestionario, sin duda han sido útiles para conocer determinados aspectos de la CA de los MFI en relación a los problemas tratados. Sin embargo, no queda tan claro que el JR produzca una mejora de los mismos. Entre Pre y Pos, la importancia que dan al problema (P12) y su predisposición a asistir a eventos informativos y formativos (P13) es la misma. La P15 del Pre ofreció datos acerca de las prácticas habituales de los MFI en cuanto al consumo y uso energético y pudo constatarse que el conocimiento general del grupo sobre buenas prácticas y alternativas de consumo se amplió (P15 del Pos).

No obstante, otros estudios sí han encontrado cambios en las actitudes y valores del alumnado tras su participación en un JR. Por ejemplo, Hernández (2010) concluía en un estudio acerca de las actitudes y valores con 396 jóvenes de nivel educativo de $2^{\underline{o}}$ de la E.S.O., en torno al autoconsumo energético, que la utilización del JR potencia los cambios en las actitudes y valores de los participantes en los mismos. España (2008) encontraba que las actitudes eran los componentes afectivos que con más abundancia y variedad se mostraban en los argumentos del alumnado al participar en un JR acerca de los alimentos transgénicos. Estos resultados muestran que sería interesante, no solo el análisis de la posible influencia sobre las actitudes que pueda tener la participación en un JR, sino también el estudio de la influencia de dichas actitudes en los argumentos elaborados por el alumnado. Por último, y en relación a la formación inicial del profesorado, Rueda (2018) encontraba que la adquisición de actitudes y valores es una de las ventajas educativas del JR más consideradas por el futuro profesorado de ciencias de secundaria, por ello, se plantea para futuros trabajos el análisis de las actitudes del profesorado en formación inicial a través de sus argumentos.

Por último, analizando de forma conjunta los resultados de P12, P14 y P16, puede verse una muestra de información concerniente a las dimensiones afectiva, actitudinal y activa, respectivamente. Los resultados obtenidos en las mismas se asemejan a una inconsistencia recurrente en la relación actitud-comportamiento (Muñoz, 2011), en lo que a CA se refiere: la existencia de disposiciones afectivas y actitudinales proambientales, no mantiene en todos los casos una relación directamente proporcional con la existencia de comportamientos proambientales, o lo que es lo mismo, la actitud no siempre determina la conducta.

\section{Agradecimientos}

Este trabajo forma parte del proyecto de I+D de Excelencia "Desarrollo de competencias en problemas de la vida diaria mediante prácticas científicas de argumentación, indagación y modelización en enseñanza secundaria y universitaria" (EDU2017-82197-P), financiado por el Ministerio de Ciencia, Innovación y Universidades en 2017. 


\section{Referencias bibliográficas}

Abella, V., y Grande, M. (2010). Juegos de rol como estrategia educativa: Percepciones de docentes en formación y estudiantes de secundaria. Teoría de la Educación. Educación y Cultura en la Sociedad de la Información, 11(3), 27-54.

Acebal, M. C. (2010). Conciencia Ambiental y Formación de Maestras y Maestros. Tesis doctoral. Universidad de Málaga, Málaga.

Barab, S., y Squire, K. (2004). Design-Based Research: putting a stake in the ground. The Journal of the Learning Sciences, 13(1), 1-14.

Cebrián, D., España, E., y Franco. A. J. (2018). Diseño de un juego de rol sobre un problema socio-científico relacionado con las centrales nucleares para iniciar en el activismo y en el uso de pruebas a maestros de primaria en formación inicial. Actas de los XXVIII Encuentros de Didáctica de las Ciencias Experimentales, 1241-1246. La Coruña: Universidad de A Coruña.

Cervilla, P. (2 de agosto de 2017). La presión política obliga al Gobierno a cerrar la central nuclear de Garoña. $A B C$. Recuperado de https://www.abc.es/sociedad/abci-presion-politica-obliga-gobierno-cerrarcentral-nuclear-garona-201708020314_noticia.html

Cobb, P., diSessa, A., Lehrer, R., y Schauble, L. (2003). Design experiments in educational research. Educational Researcher, 32(1), 9-13.

Colucci, L., Camino, E., Barbiero, G., y Gray, D. (2006). From Scientific Literacy to Sustainability Literacy: An Ecological Framework for Education. Science Education, 90, 227-252.

Creswell, J. (2005). Educational research: Planning, conducting, and evaluating quantitative and qualitative research. Upper Saddle River: Pearson Education.

De Benito, B., y Salinas, J. M. (2016). La Investigación Basada en el Diseño en Tecnología Educativa. Revista Interuniversitaria de Investigación en Tecnología Educativa, RIITE (0), 44-59. DOI: 10.6018/riite2016/260631.

Echeverría, P. (2010). El papel de la docencia universitaria en la formación inicial de profesores. Calidad en la Educación, 32, 149-165.

España, E. (2008). Conocimiento, actitudes, creencias y valores en los argumentos sobre un tema socio-científico relacionado con los alimentos. Tesis doctoral. Málaga: Universidad de Málaga.

España, E., y Prieto, T. (2010). Los problemas socio-científicos como contexto para la enseñanza y el aprendizaje de las ciencias. Investigación en la Escuela, 71, 1724.

España, E., Rueda, J. A., y Blanco, A. (2013). Juegos de rol sobre el calentamiento global. Actividades de enseñanza realizadas por estudiantes de ciencias del Máster en Profesorado de Secundaria. Revista Eureka de Enseñanza y Divulgación de las Ciencias, 10 (nº extraordinario), 763-779.

Gallego, D., Bustamante, L., Gallego, L., Salcedo, L., Gava, M., y Alfaro, E. (2017). Estudio cuantitativo sobre las concepciones de ciencia, metodología y enseñanza para profesores en formación. Revista Lasallista de Investigación, 4(1), 144-161. 
García, D., Castillo, C., Rios, S., Cristofol, C., Carrasco, M. J., Rodríguez, R. M., Pastor, I., y González, D. (2011). La interdisciplinariedad en la educación superior: propuesta de una guía para el diseño de juego de rol. TESI, Teoría de la Educación: Educación y Cultura en la Sociedad de la Información, 12(1), 386413.

García, E. M., González, J. C., López, J. A., Luján, J. L., Martín, M., Osorio, C., y Valdés, C. (2001). Ciencia, Tecnología y Sociedad: una aproximación conceptual. Madrid, España: Organización de Estados Iberoamericanos para la Educación, la Ciencia y la Cultura.

Gomera, A., Villamandos, F., y Vaquero, M. (2012). Medición y categorización de la conciencia ambiental del alumnado universitario: contribución de la universidad a su fortalecimiento. Profesorado. Revista de Currículum y Formación de Profesorado, 16(2), 193-212.

Hernández, J. (2010). Cambio de actitudes y valores ante la energía tras el uso de un juego de rol. Teoría de la Educación. Educación y Cultura en la Sociedad de la Información, 11(3), 135-148.

Hodson, D. (2003). Time for action. Science education for an alternative future. International Journal of Science Education, 25(6), 645-670.

Howes, E. V., y Cruz, B. C. (2009). Role-playing in science education: an effective strategy for developing multiple perspectives. Journal of Elementary Science Education, 21(3), 33-46

Jiménez-Aleixandre, M. P. (2010). 10 ideas clave. Competencias en argumentación y uso de pruebas. Barcelona: Graó.

Jiménez, M., y Lafuente, R. (2006). La operacionalización del concepto de conciencia ambiental en las encuestas. La experiencia del Ecobarómetro andaluz. En R. de Castro (Coord.), Persona, Sociedad y Medio Ambiente. Perspectivas de la investigación social de la sostenibilidad (pp. 121-150). Sevilla, España: Junta de Andalucía.

Juárez, P., Hierrezuelo, J. M., Cebrián, D., y Franco, A. J. (2019). El juego de rol como estrategia para enseñar a argumentar en ciencias. La visión de maestros en formación inicial. Aula de Innovación Educativa, 287, 15-20.

Karahan, E., y Roehrig, G. (2015). Constructing media artefacts in a social constructivist environment to enhance students' environmental awareness and activism. Journal of Science Education and Technology, 24, 103-118.

Lacave, C., Molina, A. I., Fernández, M., y Redondo, M. A. (2016). Análisis de la fiabilidad y validez de un cuestionario docente. Visión, Revista de Investigación en Docencia Universitaria de la Informática, 9(1), 23-36.

Mansour, N. (2009). Science Teachers' Beliefs and Practices: Issues, Implications and Research Agenda. International Journal of Environmental \& Science Education, $4(1), 25-48$.

Martín, C., Prieto, T., y Jiménez, M. A. (2013). El problema de la producción y el consumo de energía: ¿cómo es tratado en los libros de texto de educación secundaria? Enseñanza de las Ciencias, 31(2), 153-171. 
Matas, A. (2003). Los juegos de rol como recurso formativo. Una aplicación en educación ambiental. Bordón. Revista de pedagogía. 55(2), 281-291.

Matas, A. (2008). Los juegos de rol. Un acercamiento psicopedagógico. Málaga: Adiesoc.

McSharry, G., y Jones, S. (2000). Role-play in science teaching and learning. School Science Review, 82(298), 73-82.

Meinhold, J. L., y Malkus, A. J. (2005). Adolescent environmental behaviors: can knowledge, attitudes, and self-efficacy make a difference? Environmentand behavior, 37, 511-532.

Muñoz, A. (2011). Concepto, expresión y dimensiones de la conciencia ambiental. El papel de la cultura científica. Saarbrücken, Alemania: Editorial Académica Española.

Puig, B., y Evagorou, M. (2020). Design of a socioscientific issue unit with the use of modelling: the case of bees. International Journal of Designs for Learning, 11(1), 98-107.

Rojas, M. J., y Zapata, P. N. (2017). Contribuciones de la práctica pedagógica a la construcción del conocimiento profesional de profesores de ciencias en formación inicial. En Actas X Congreso Internacional sobre Investigación en Didáctica de las Ciencias, Enseñanza de las Ciencias, no extraordinario, 413-417. https://ddd.uab.cat/record/184620?ln=ca.

Romero, M. (2014). Uniendo investigación, política y práctica educativas: DBR, desafíos y oportunidades. Magis. Revistas Internacional de Investigación en Educación, 7(14), 159-176.

RTVE (2017). Greenpeace vaticina «el fin de la energía nuclear en España» con el cierre de Garoña. (1 de agosto de 2017). RTVE. Recuperado de http://www.rtve.es/noticias/20190306/grandes-electricas-pactan-cierreescalonado-centrales-nucleares-espanolas-entre-2025-2035/1896202.shtml.

RTVE (2019). Las grandes eléctricas pactan el cierre escalonado de las centrales nucleares españolas entre 2025 y 2035. (6 de marzo de 2019). RTVE. Recuperado de http://www.rtve.es/noticias/20190306/grandes-electricaspactan-cierre-escalonado-centrales-nucleares-espanolas-entre-20252035/1896202.shtml.

Rueda, J. A. (2018). Las actividades de juego de rol en el marco de una propuesta en la formación inicial del profesorado de ciencias de educación secundaria contextualizada en el problema del calentamiento global. Tesis doctoral. Málaga: Universidad de Málaga.

Sadler, T. D. (2011). Socio-scientific Issues in the Classroom: Teaching, Learning and Research. London (U. K.): Springer

Sauvé, L. (2010). Educación científica y educación ambiental: un cruce fecundo. Investigación Didáctica, 28(1), 5-18.

Schein, E. H. (1988). La cultura empresarial y el liderazgo. Una visión dinámica. Barcelona: Plaza y Janés. 
Simonneaux, L. (2000). Cómo favorecer la argumentación sobre las biotecnologías entre el alumnado. Alambique. Didáctica de las Ciencias Experimentales, 25, 2744.

Simonneaux, L. (2001). Role-play or debate to promote students' argumentation and justification on an issue in animal transgenesis. International Journal of Science Education, 23(9), 903-927.

Simoneaux, L. (2008). Argumentation in Socio-Scientific Contexts. En S. Erduran y M. Jiménez-Aleixandre. (Eds.), Argumentation in science education: perspectives from classroom-based research (179-199). Dordrecht, Netherlands: Springer.

Tójar, J. C. (2006). Investigación Cualitativa. Comprender y actuar. Madrid: La Muralla.

Valbuena, E. O. (2007). El conocimiento didáctico del contenido biológico: estudio de las concepciones disciplinares y didácticas de futuros docentes de la Universidad Pedagógica Nacional (Colombia). Tesis doctoral. Madrid: Universidad Complutense de Madrid.

Vázquez, A., y Manassero, M. A. (2008). El declive de las actitudes hacia la ciencia de los estudiantes: un indicador inquietante para la educación científica. Revista Eureka de Enseñanza y Divulgación de las Ciencias, 5(3), 274-292.

Villa, L. (2016). Garoña marca el futuro de la energía nuclear en España. Público. Recuperado de https://www.publico.es/politica/garona-marca-futuroenergia-nuclear.html. 


\section{Anexo I}

\section{Pretest}

1. Imagina que eres un diputado y puedes votar para cambiar las leyes. Garoña es una central nuclear de 45 años y el periodo de vida útil que tienen las centrales nucleares es de 40 años, es decir, para que siga funcionando habría que cambiar la ley, ¿Estarías a favor de cambiar dicha ley y ampliar a 50/60 años la vida útil de las centrales?

2. Justifica la elección que tomaste de cambiar la ley. ¿Por qué consideras importante cambiar o no la ley?

3. El gas que sale de las centrales nucleares es:
a. $\mathrm{CO} 2$
d. Aire
b. Helio
e. No hay gases que salen
c. Agua
f. Contaminantes varios

4. Enumera las ventajas que tiene la energía nuclear para ti (Ejemplo: 1. Es barata 2. Es útil para España...)

5. En una central nuclear española el proceso que se hace se llama:
a. Reacción nuclear
c. Fusión nuclear
b. Fisión nuclear
d. No sé

6. Enumera los inconvenientes que conozcas que presenta la energía nuclear (Ejemplo: 1 . Es cara 2. No nos sirve en España...)

7. ¿Qué se hace con los residuos de las centrales nucleares en España?
a. Se vierten al mar
b. No hay residuos sólidos
c. Se entierran bajo tierra

d. Se entierran bajo tierra, pero se guardan dentro de paredes de hormigón

e. Se llevan a otros países para que lo gestionen

8. ¿Conoces el nombre de alguna central nuclear española?

9. Describe brevemente cómo se obtiene energía en la central nuclear

\section{Postest}

Preguntas de la 1 a la 9

10. ¿Crees que los juegos de rol son interesantes para trabajarlos en primaria?

11. ¿Por qué elegiste la opción anterior que trataba sobre si eran interesantes los juegos de rol en primaria? 


\section{Pretest}

\section{Anexo II}

1. ¿Crees que se debería cesar el uso de energía nuclear en España para el 2020?

2. Argumenta todo lo mejor que puedas tu decisión anterior

3. El gas que sale de las centrales nucleares es:
a) Dióxido de
b) Helio
d) Aire
f) No salen gases carbono
c) Vapor de agua
e) Otros gases
g) No sé

4. Enumera las ventajas que tiene la energía nuclear para ti

5. Enumera los inconvenientes que tiene la energía nuclear para ti

6. En una central nuclear el proceso de obtención de calor se produce mediante
a) Fusión nuclear
c) Evaporación de agua
e) Combustión
b) Reacción nuclear
d) Fisión nuclear
f) No sé

7. ¿Qué crees que se suele hacer con los residuos sólidos de las centrales nucleares?
a) Se vierten al mar
d) Se entierran bajo tierra, pero se guardan
b) No hay residuos sólidos dentro de paredes de hormigón
c) Se entierran bajo tierra
e) Se llevan a otros países para que lo gestionen

8. Justifica por qué has elegido esa opción

9. Describe todo el proceso de obtención de energía en una central nuclear

10. ¿Crees que los juegos de rol son actividades interesantes para trabajarlos en primaria?
a. Sí
c. Depende
b. No
d. No sé

11. Justifica la opción anterior

\section{Postest}

Preguntas de la 1 a la 11

12. Justifica la opción anterior

13. ¿Qué es lo que más te ha gustado de la actividad? Explica tu respuesta

14. ¿Qué es lo que menos te ha gustado de la actividad? Explica tu respuesta

15. ¿Qué cambiarías en la actividad? Explica tus respuestas

16. Expresa tu grado de acuerdo, 1 (total desacuerdo) a 5 (total acuerdo), con las siguientes afirmaciones sobre el juego de rol que acabas de hacer. Hay preguntas en negativo, lee con atención
a) La actividad que hemos desarrollado en clase es útil para mí como docente para el futuro profesional de mis estudiantes
f) La actividad es inútil
b) La actividad que hemos desarrollado en clase es útil para mis estudiantes
g) La actividad es adecuada para el desarrollo de competencias
c) La actividad no es útil para motivar a mis estudiantes
h) $\mathrm{Me}$ ha gustado la actividad que acabamos de desarrollar
d) La actividad es inviable para mis futuras clases
e) La metodología seguida es útil para el trabajo colaborativo
i) La actividad que acabamos de desarrollar se hizo larga
j) Me ha gustado cómo se planteó la evaluación de la actividad
k) La actividad es clara $y$ fácil de entender.

l) La actividad fue compleja de elaborar

m) Esta actividad y la metodología mejoró mi motivación hacia el tema o tarea

n) Se han visto mejoradas mis competencias con la actividad

o) La actividad no es innovadora para el contexto de mis futuras clases

17. ¿Algo más que aportar? Incluye aquí cualquier otro comentario que quieras hacer sobre la actividad del juego de rol. 


\section{Anexo III}

Pretest

1. ¿Estás de acuerdo con el pacto de cierre de las centrales nucleares españolas entre los años $2030 \mathrm{y}$ 2035?

a. Sí, estoy de acuerdo con el pacto realizado de cese del uso de energía nuclear entre los años 20302035

b. No estoy de acuerdo con el pacto, creo que debería mantenerse el uso de energía nuclear por tiempo indefinido

c. No estoy de acuerdo con el pacto, debería de eliminarse en el año 2020 el uso de energía nuclear

2. Justifica la respuesta anterior

3. Explica si estarías de acuerdo o no con la instalación de una central nuclear en tu municipio de residencia. ¿Y con la instalación de un cementerio nuclear?

a. Estaría de acuerdo con la instalación de una c. Estaría de acuerdo con la instalación de central nuclear ambas

b. Estaría de acuerdo con la instalación de un d. No estaría de acuerdo con la instalación de cementerio nuclear

no estaria

4. ¿Qué diferencia piensas que hay entre una central nuclear y un cementerio nuclear?

5. Describe, de principio a fin, todo el proceso que piensas que se lleva a cabo en una central nuclear.

6. Enumera las principales ventajas que crees que tiene el uso de energía nuclear ( 1 o 2)

7. Enumera las principales desventajas que crees que tiene el uso de energía nuclear $\left(\begin{array}{lll}1 & \circ & 2\end{array}\right)$

8. ¿Cómo se llama el proceso de obtención de calor que se llevan a cabo en una central nuclear?
a. Fusión nuclear
c. Fisión nuclear
b. Reacción nuclear
d. Evaporación de agua
e. Combustión
f. No lo sé

9. ¿Qué gas expulsan las centrales nucleares?
a. Dióxido de carbono
c. Vapor de agua
e. No lo sé
b. Helio
d. No expulsan gases

10. ¿Qué piensas que se hace con los residuos sólidos producidos en las centrales nucleares?
Se vierten al mar
c. Se entierran bajo tierra
b. No se generan residuos sólidos en las centrales
d. Se llevan a otros países
e. Se entierran bajo tierra, para que los gestionen pero se guardan dentro de paredes de hormigón nucleares

f. No lo sé

11. ¿Piensas que actualmente en España tenemos algún problema relacionado con la energía? Explica tu respuesta, y si es sí, concreta cuál/es problema/s.

12. ¿Crees que es importante informarse sobre el tipo de fuentes de energía de las que proviene nuestro consumo cotidiano? a) Sí $\quad$ b) No

13. Explica brevemente tu elección anterior

14. ¿Estarías dispuesto/a a asistir a algún evento (charlas, conferencias, foros, organizaciones, cursos o

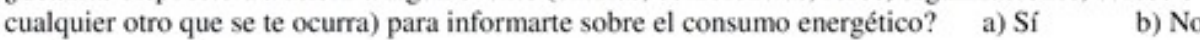

15. ¿Contribuyes o intentas contribuir con tus conductas habituales para hacer un uso responsable de la energía? Si es así, ¿de qué forma?

16. ¿Participas o has participado en eventos (charlas, conferencias, blogs, foros, organizaciones, asociaciones, manifestaciones o por cualquier otro medio que se te ocurra), para informarte sobre el consumo energético? Sí es así, menciona brevemente qué tipo de evento fue.

\section{Postest}

Preguntas de la 1 a la 14

15. Durante la preparación del juego de rol, ¿has descubierto o pensado medidas con las que podrías contribuir con tus conductas habituales para hacer un uso responsable de la energía? Si es así. ¿cuál/es?

16. ¿Consideras el juego de rol un buen recurso para utilizarlo en la educación primaria? Explica por qué sí o por qué no.

17. ¿Consideras adecuado tratar problemas ambientales mediante juegos de rol en educación primaria? $\mathrm{Si}$ es así, explica por qué y con qué problema/s lo harías. 